\title{
Assessing direct healthcare costs when restricted to self-reported data: a scoping review
}

Samira B. Jabakhanji ${ }^{1} \mathbb{D}$, Jan Sorensen ${ }^{1}$ (D), Gintare Valentelyte ${ }^{1}$ (D), Lee Ann Burke ${ }^{2}$, Brendan McElroy ${ }^{2}$ (D) and Aileen Murphy ${ }^{2^{*}}$ (D)

\begin{abstract}
Background: In the absence of electronic health records, analysis of direct healthcare costs often relies on resource utilisation data collected from patient-reported surveys. This scoping review explored the availability, use and methodological details of self-reported healthcare service utilisation and cost data to assess healthcare costs in Ireland.

Methods: Population health surveys were identified from Irish data repositories and details were collated in an inventory to inform the literature search. Irish cost studies published in peer-reviewed and grey sources from 2009 to 2019 were included if they used self-reported data on healthcare utilisation or cost. Two independent researchers extracted studies' details and the PRISMA-ScR guidelines were used for reporting.

Results: In total, 27 surveys were identified containing varying details of healthcare utilisation/cost, health status, demographic characteristics and health-related risk and behaviour. Of those surveys, 21 were general population surveys and six were study-specific ad-hoc surveys. Furthermore, 14 cost studies were identified which used retrospective self-reported data on healthcare utilisation or cost from ten of the identified surveys. Nine of these cost studies used ad-hoc surveys and five used data from pre-existing population surveys. Compared to population surveys, ad-hoc surveys contained more detailed information on resource use, albeit with smaller sample sizes. Recall periods ranged from 1 week for frequently used services to 1 year for rarer service use, or longer for once-off costs. A range of perspectives (societal, healthcare and public sector) and costing approaches (bottom-up costing and a mix of top-down and bottom-up) were used. The majority of studies ( $n=11)$ determined unit prices using multiple sources, including national healthcare tariffs, literature and expert views. Moreover, most studies $(n=13)$ reported limitations concerning data availability, risk of bias and generalisability. Various sampling, data collection and analysis strategies were employed to minimise these.
\end{abstract}

Conclusion: Population surveys can aid cost assessments in jurisdictions that lack electronic health records, unique patient identifiers and data interoperability. To increase utilisation, researchers wanting to conduct cost analyses need to be aware of and have access to existing data sources. Future population surveys should be designed to address reported limitations and capture comprehensive health-related, demographic and resource use data.

Keywords: Costs, Resources, Surveys, Scoping review, Ireland

\footnotetext{
* Correspondence: aileen.murphy@ucc.ie

${ }^{2}$ Department of Economics, Cork University Business School, University College Cork, Cork, County Cork, Ireland

Full list of author information is available at the end of the article
}

(c) The Author(s). 2021 Open Access This article is licensed under a Creative Commons Attribution 4.0 International License, which permits use, sharing, adaptation, distribution and reproduction in any medium or format, as long as you give appropriate credit to the original author(s) and the source, provide a link to the Creative Commons licence, and indicate if changes were made. The images or other third party material in this article are included in the article's Creative Commons licence, unless indicated otherwise in a credit line to the material. If material is not included in the article's Creative Commons licence and your intended use is not permitted by statutory regulation or exceeds the permitted use, you will need to obtain permission directly from the copyright holder. To view a copy of this licence, visit http://creativecommons.org/licenses/by/4.0/. The Creative Commons Public Domain Dedication waiver (http://creativecommons.org/publicdomain/zero/1.0/) applies to the data made available in this article, unless otherwise stated in a credit line to the data. 


\section{Introduction}

In the absence of electronic health records (EHR) linkable through unique patient identifiers, researchers must rely on collecting or using secondary healthcare-user data to assess healthcare utilisation and cost. This is the case in Ireland, which is one of seven Organisations for Economic Co-operation and Development countries that lack a comprehensive system of unique patient and provider identifiers [1]. Despite efforts to roll out Individual Health Identifiers and implement EHRs, data are not regularly captured in national statistics monitoring health system performance, patient safety or public health. Overall, employed systems lack interoperability, change is slow and often data are fragmented and records inaccessible [1-3], meaning that records in Ireland cannot be linked, yielding barriers to fully developing and using top-down costing approaches. Accordingly, it is difficult to obtain comprehensive descriptions of cost and resource use at a local or national level to inform policy and practice planning to prioritise prevention and match patient needs. While these barriers are not unique to Ireland they rarely occur cumulatively [1]. Consequential resource allocation decisions can lead to suboptimal patient care [4].

Cost analyses of healthcare resources provide information about current allocation and inform debates about rational and efficient future redistribution of resources for prevention and treatment of ill-health, complementing clinical evidence in cost-effectiveness analyses for example where used [5]. Reliable and detailed data could facilitate tailored resource allocations to individual patient and population groups, based on which the staffing and funding of healthcare services can be organised to meet current and future healthcare demands. This requires the identification of health threats, healthcare need and cost drivers of healthcare in the first place, all of which can then be addressed through intervention studies (trials) to identify cost-effective strategies for health policy and clinical practice.

For planning and evaluation, it is common practice to use the principle of attributable costs to establish the costs related to risk factors or long-term cost of a disease. The attributable cost approach requires that the cost of healthcare provided to two groups of individuals can be established by aggregating different sources of data about resource use and cost for each individual [6]. To attribute costs, data are needed in relation to the individual health status (e.g. disease history and outcomes; anthropometric measures; laboratory data; quality of life) and healthcare utilisation (e.g. type, frequency and time of health service use). For a comprehensive identification of cost drivers and needs, additional data requirements include demographic information (sex; age), information on health-related risk and behaviour (genetic predisposition; socio-economic status; environmental factors; lifestyle) and, to integrate a societal perspective, data on labour force participation (absenteeism; presenteeism) and opportunities (educational outcomes) are needed. To maximise accuracy, these various data from an individual should be linked, and available over time. Ideal sources include patient record files and (diseasespecific) national registries containing additional details on health-related risk factors, behaviours, and labour force participation [7].

While method guides exist for some jurisdictions, primarily for conducting health economic evaluations, inconsistencies persist with different costing methods and approaches yielding different results. In response, there are calls for more consistent and efficient costing methods [5]. Generally, health system costs can be assessed using top-down approaches (gross costing) which rely on centralised data repositories, such as hospital patient record files collected prospectively. Alternatively bottom-up approaches (micro or activity-based costing) using data collected directly from healthcare users, often through surveys, censuses or diaries. The advantages of top-down costing include the requirement of fewer resources and provision of better opportunities for generalisation, but at the expense of precision when informing economic evaluations of interventions. Bottom-up costing is more laborious, but provides greater insights into the relationship between activity and cost characteristics, the economies of scale, and the relative costs of different activities and variation in patient-related cost $[8,9]$.

Thus, when analysing data on healthcare utilisation in jurisdictions without HER or linked systems, like Ireland, researchers have limited options. Individual data may be collected from 'top-down' perspective from individual service providers, incurring a high administrative and resource burden. Alternatively, self-reported user data may be used. Often this includes cross-sectional and longitudinal quantitative data collected from general population health surveys or disease-specific surveys and extrapolated to population level, from a 'bottom-up' approach. In comparison, trial data from intervention studies typically involve small disease- and location-specific populations and are less representative of and generalisable to regional or national populations. While data on healthcare utilisation and cost measures gathered from quantitative surveys potentially lack precision, they frequently represent the best available data to assess healthcare costs in jurisdictions like Ireland.

While the existence of this issue is commonly acknowledged, here we explore the extent to which general population health surveys are used to overcome it when conducting cost analyses. In doing so we firstly identify the self-reported health service utilisation and cost 
surveys/datasets available, and consider their suitability for costing direct healthcare use. Secondly, we conduct a scoping review to identify and examine Irish costing studies (including cost analysis, cost estimation and cost of illness) that applied these surveys/datasets to calculate direct healthcare costs.

\section{Methods}

An inventory of Irish population health surveys was developed and a scoping review $[10,11]$ was conducted to map the methodologies and data sources used in Irish studies of direct healthcare costs.

\section{Search strategy}

Initially, the Irish Social Science Data Archive, safefood, Central Statistics Office and the Department of Health websites and repositories were searched to identify population surveys that contain data on health, healthcare utilisation or healthcare-related cost. To obtain additional information for each of the identified surveys, survey-specific websites and original questionnaires were reviewed. The list of surveys informed the subsequent literature search.

A PubMed literature search, conducted from 16th to 18th October 2019, identified studies published between October 2009 and October 2019 that assess selfreported healthcare use and cost data in the Republic of Ireland. Initially, the MeSH Term 'cost' was combined with 'questionnaire' $[\mathrm{MeSH}]$. Subsequently, 'cost' $[\mathrm{MeSH}]$ was combined with the full name and abbreviations of the identified population health surveys (Supplementary Material 1). We also searched grey literature for reports and scientific papers not listed on PubMed. Specifically, the Irish Social Science Data Archive, safefood, Central Statistics Office and the Department of Health websites and repositories were searched for healthcarerelated publications.

\section{Analysis}

For each population health survey, information was extracted on data collection tools, sample characteristics and the type of healthcare services for which data were collected. Additionally, availability of demographic information, socio-economic information, health status and lifestyle variables from surveys were recorded. This information is collated in an inventory.

For the scoping review of published studies, two researchers independently reviewed the abstracts of all retrieved citations. Initial charting included basic information of the underlying dataset and availability of data on healthcare utilisation or costs. Full-text studies and reports were retained if they (1) used survey or otherwise self-reported data on healthcare utilisation or cost, including patients' direct out-of-pocket costs, and
(2) used these data to assess direct healthcare costs. Whereby direct healthcare costs only refer to resources used in the healthcare sector (excluding resources used in other sectors, such as social care) [12]. The following exclusion criteria were applied: Publication outside the study period and region; studies reporting indirect healthcare costs only; studies using simulations, decision-trees or Markov-models only; studies applying primary data from other jurisdictions to the Irish setting; and study protocols. Intervention studies reporting treatment costs (clinical trials) were excluded as these are often not representative of the Irish population and data not always self-reported. Patient registry studies were excluded as these data are often not self-reported.

Studies qualifying for inclusion were reviewed in full and information of data sources and costing methodologies extracted. In particular, details of perspectives and costing methods were identified, areas of health services for which costing was undertaken and reflections on costing methods and datasets were synthesised. Methodological quality and risk of bias were not assessed as this not a common component of scoping reviews [10]. Findings were reported using the PRISMA-ScR guidelines $[13,14]$.

\section{Results}

\section{Survey inventory}

We identified 21 population surveys or survey tools that contain data on health status, healthcare utilisation or healthcare cost, available from Irish data repositories (Table 1). The literature review identified six additional surveys that researchers used on an ad-hoc basis to inform their assessment of direct healthcare costs (included on Table 2).

The earliest of these surveys started collecting Irish health data in the 1970s (Census of Population), and approximately half include repeated longitudinal or crosssectional data collection. Sample sizes vary, ranging from 100 participants in an ad-hoc survey (e.g. in the Enhancing Care in Alzheimer's disease (ECAD) study) to thousands of participants in the nationally representative longitudinal surveys, (e.g. Growing Up in Ireland (GUI) '98 $(n=8570)$ and '08 cohorts $(n=11,134)$; the Irish Longitudinal Study on Ageing (TILDA) cohort ( $n=$ 6279)); cross-sectional repeated surveys (Irish Health Survey $(n=10,323)$, EU-SILC-Ireland $(n=11,130)$, etc.); and national single-use surveys (All Ireland Traveller Health Study $(n=8430)$ and Irish Study of Sexual Health and Relationships $(n=7668)$ ).

Of those surveys assessing healthcare service use $(n=$ $16)$, most include data on primary care use $(n=14)$; more than half provide information on specialist services $(\mathrm{n}=11)$, medicinal products $(\mathrm{n}=10)$ or hospital outpatient service use $(n=9)$. Whereas fewer collect data on 


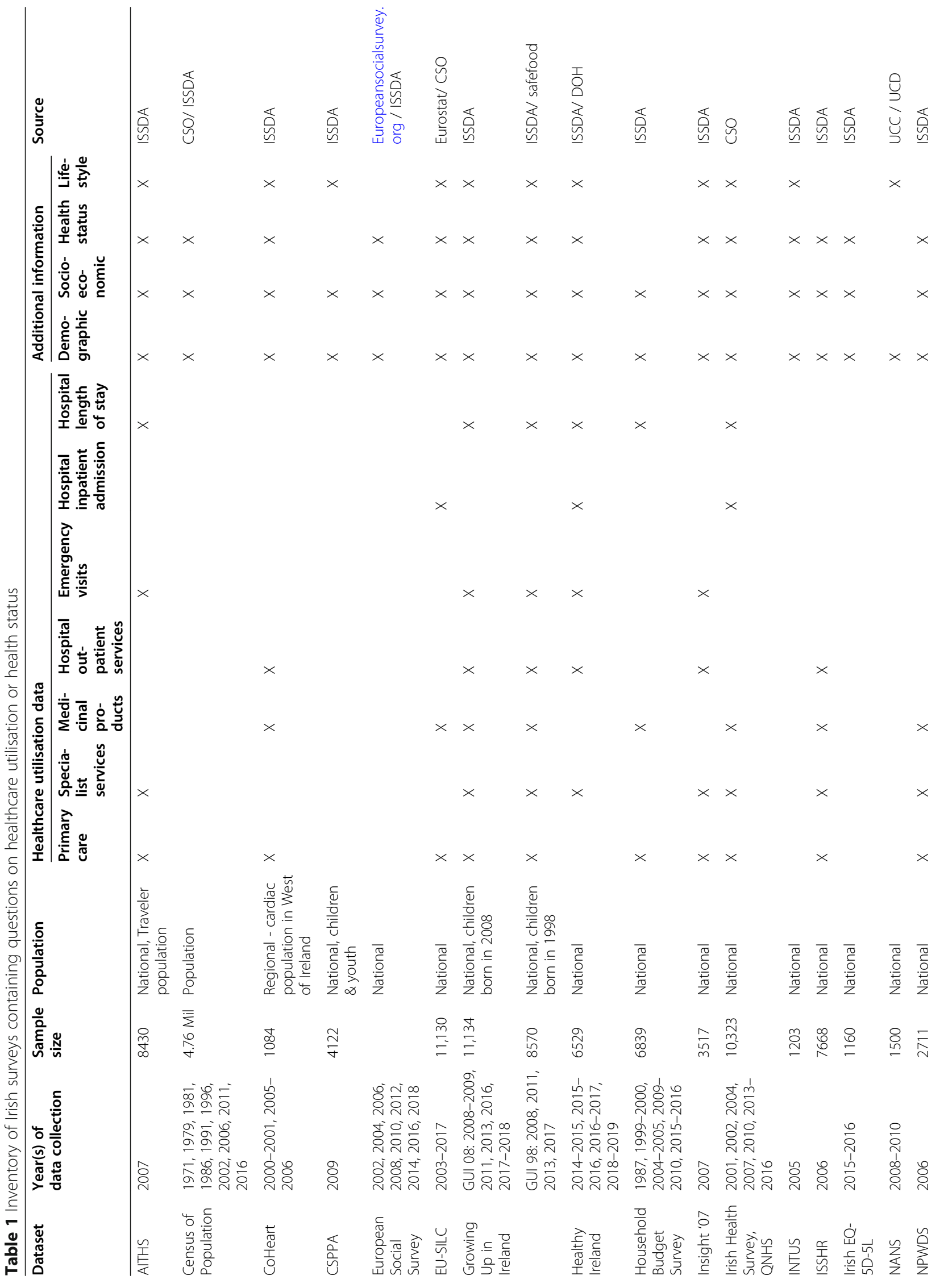




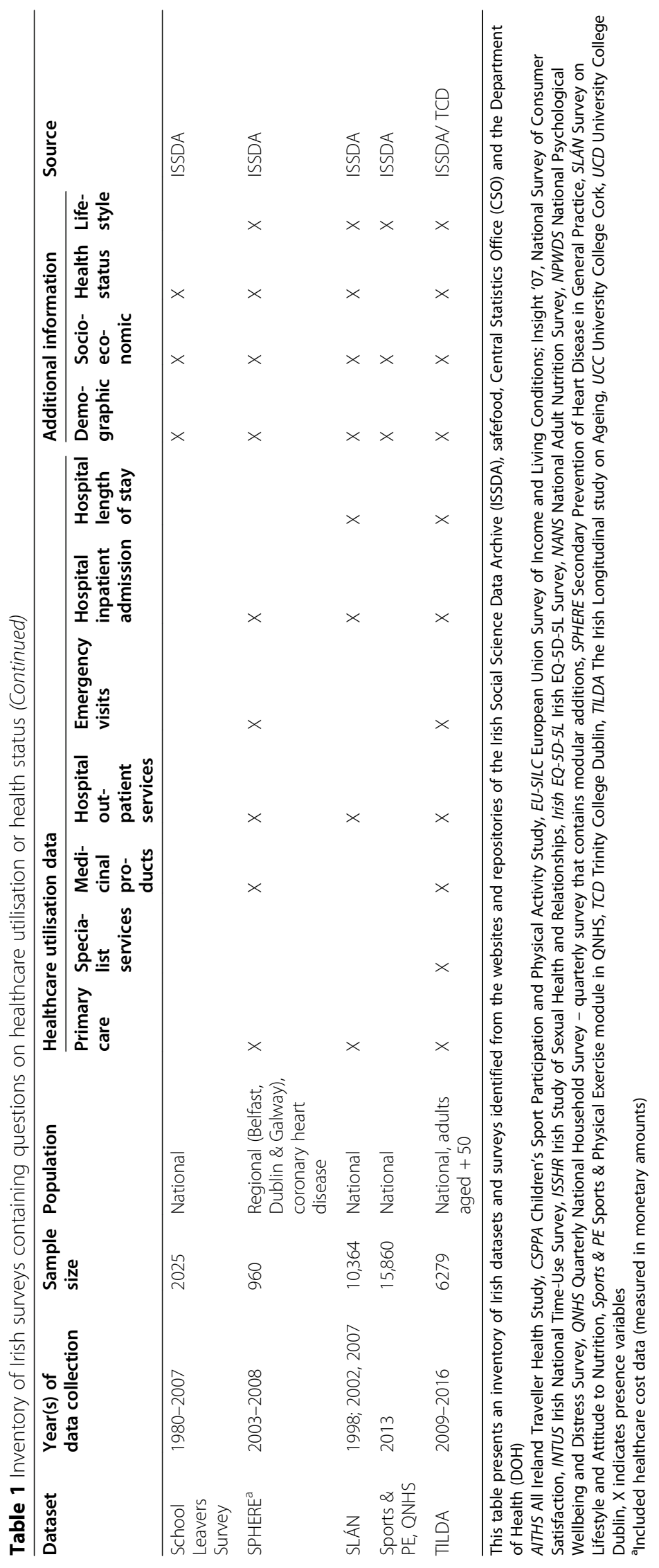




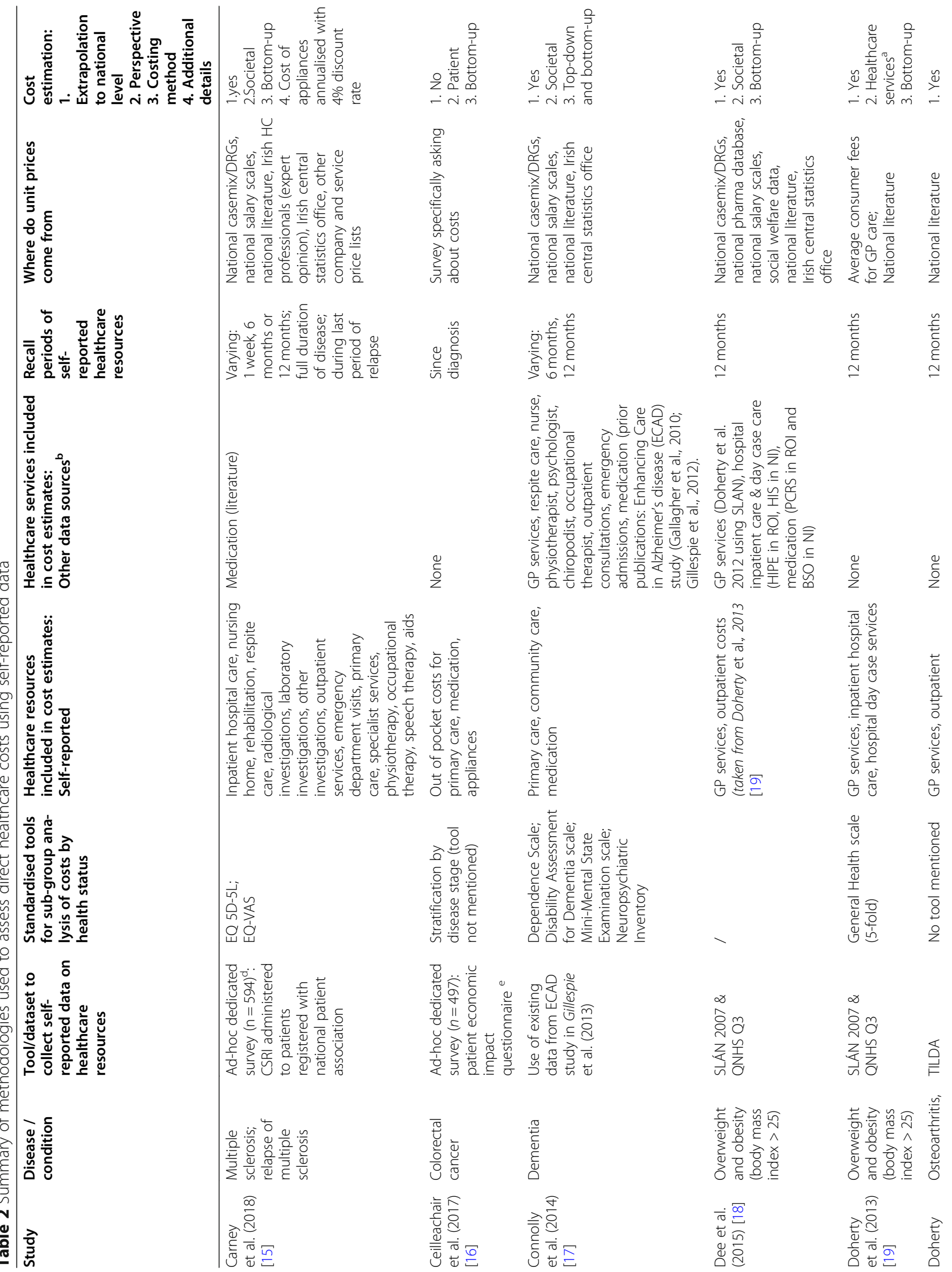




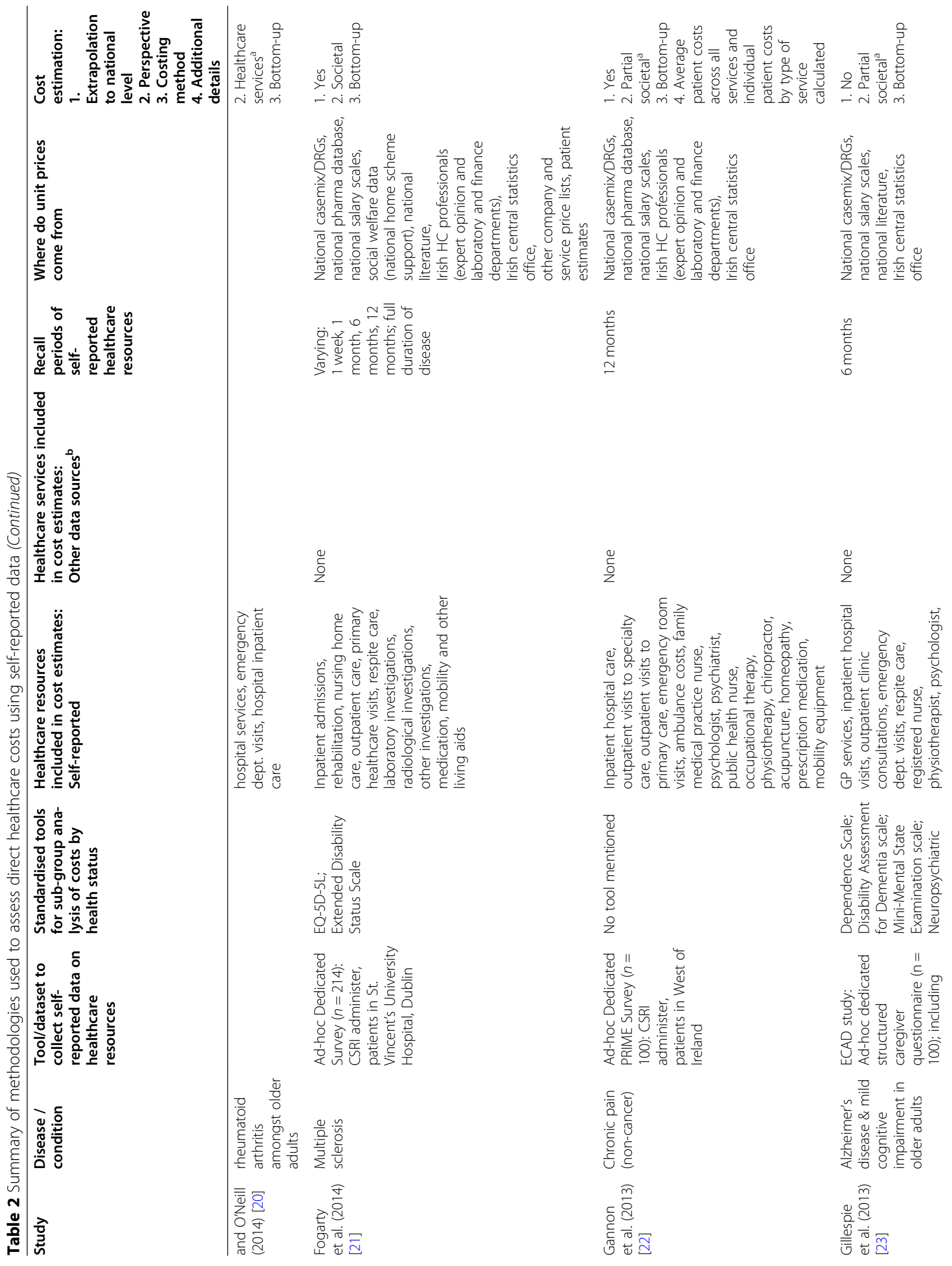




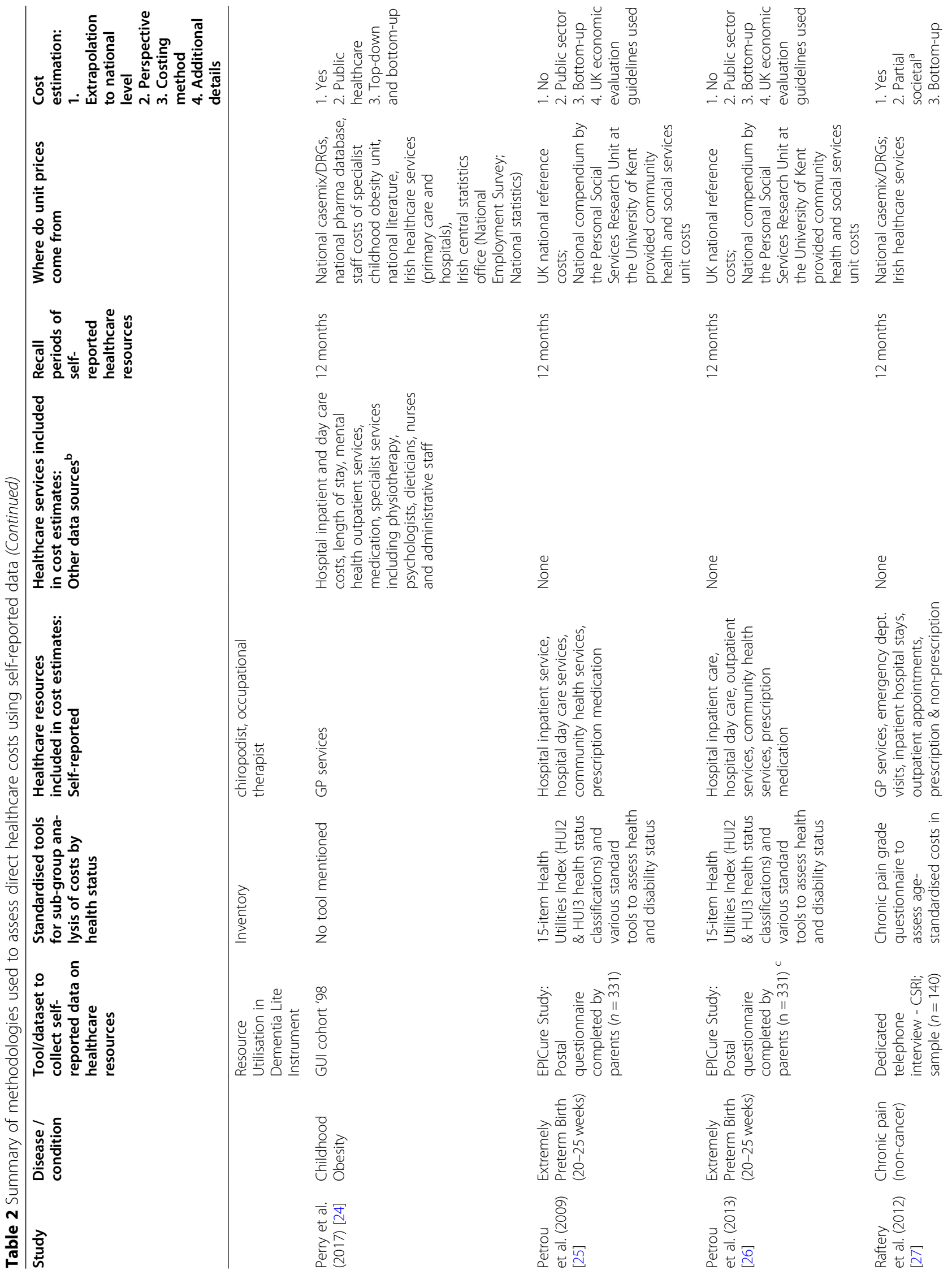




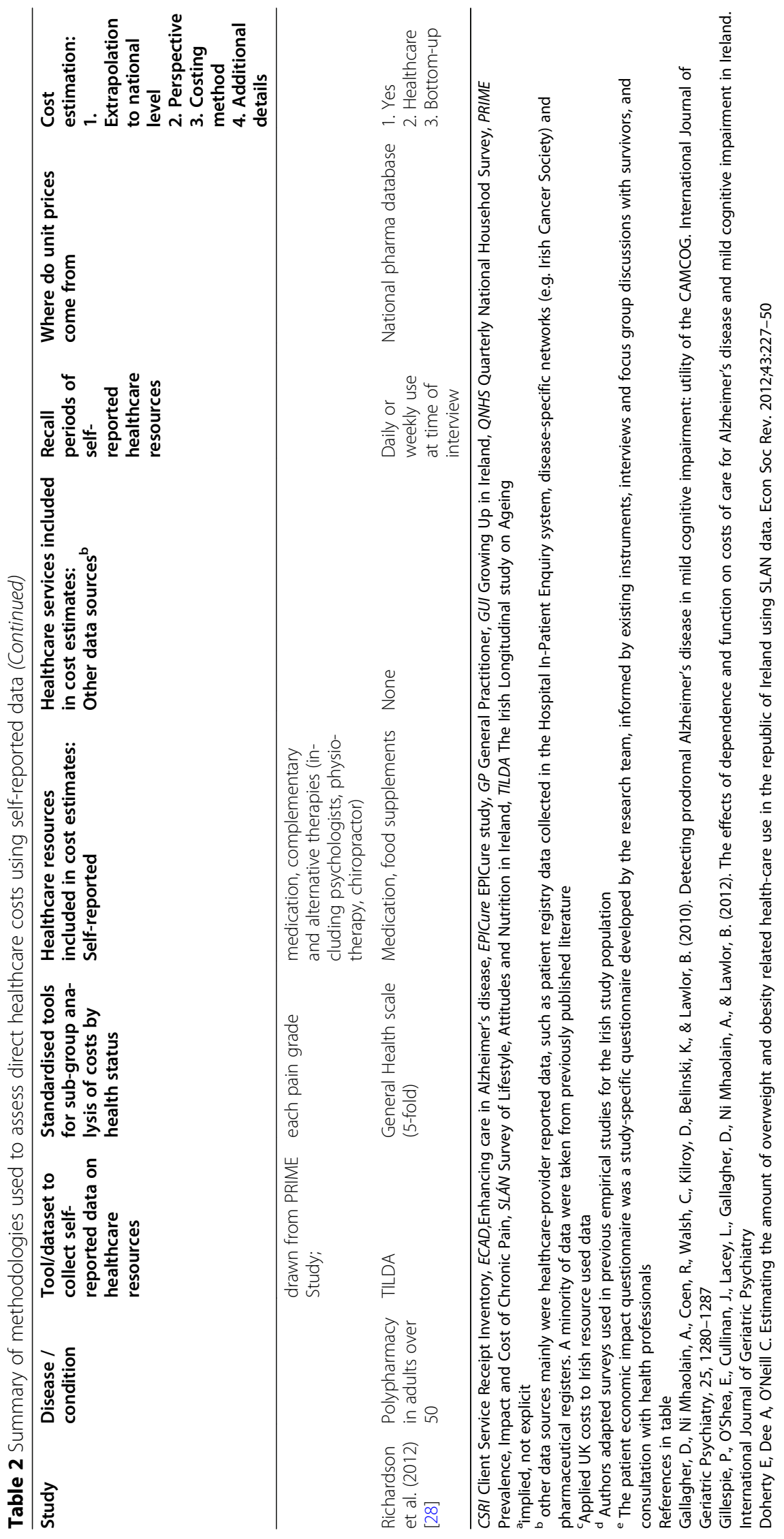


emergency department visits $(\mathrm{n}=7)$, hospital inpatient admissions $(n=6)$ and number of hospital bed days $(\mathrm{n}=$ $7)$. Almost all surveys provide demographic $(n=24)$ and socio-economic information $(n=24)$, many reported on the health status of participants $(n=19)$ and lifestyle variables $(\mathrm{n}=14)$.

\section{Scoping review}

The literature search identified 247 abstracts and reports, 165 of which were removed during initial screening and 68 were excluded after full-text review. (For a full list of search terms and results and an overview of the screening process see Supplementary Materials 1 and 2.) After applying the eligibility criteria, 11 peerreviewed publications and two reports were retained [15-18, 20-28]. Furthermore, one additional study was included from cross-referencing [19].

\section{Summary of studies}

The reviewed full-texts cover the full search period (2009-2018) across a range of eight conditions. Of the 27 surveys identified, 10 are utilised in the following studies.

Fogarty et al. (2014) examine direct and indirect 2012costs (various perspectives) related to Multiple Sclerosis, three disease subtypes and associated disability categories, in 214 patients sampled from a specialist outpatient clinic in Ireland's capital Dublin. Additionally, Carney et al. (2018) measure 2015-societal costs (direct, indirect, intangible and relapse costs) of multiple sclerosis in a nationally representative sample of multiple sclerosis patients $(n=594)$ sampled from a patient registry and through national press and online media.

Ceilleachair et al. (2017) investigate various direct and indirect out-of-pocket-costs (2008) in 497 colorectal cancer survivors identified through the National Cancer Registry from their initial diagnosis through treatment and including their initial follow-up approximately 1 year after diagnosis.

Gillespie et al. (2013) study the formal and informal cost of care (2008) related to patient dependence and function in patients with Alzheimer's disease and amnestic mild cognitive impairment, using data from the ECAD study that were collected from the caregivers of 100 community dwelling dementia patients in a memory clinic in Dublin. Furthermore, Connolly et al. (2014) assess the costs of dementia in 2010 related to informal care, health and social care, long-stay care and mortality, performing secondary analysis of the ECAD study data and merging this with (unlinked) national hospital and psychiatric inpatient data.

Accounting for the relationship between weight status and various high-cost health conditions, Doherty et al. (2013) estimate the direct healthcare costs (primary care, inpatient hospital care and day care) associated with adult overweight and obesity in Ireland in 2007 using data from 9728 adults in the nationally representative Survey on Lifestyle And Nutrition (SLAN 2007) and Quarterly National Household Survey (QNHS) Q3 2007 $(n=21,253)$. Dee et al. (2015) combine top-down and bottom-up cost analysis to estimate the full direct and indirect costs (healthcare, medication, productivity losses and mortality) related to adult overweight and obesity in Northern Ireland and Ireland in 2007, integrating the costs of co-morbidities attributable to weight status. Underlying data include the findings from Doherty et al. (2013), cross-sectional, nationally representative data from TILDA, national public hospital and reimbursement data and publicly available mortality statistics. Moreover, Perry et al. (2017) estimate the 2015-direct healthcare costs and projected indirect societal lifetime costs of childhood overweight and obesity in Northern Ireland and Ireland. The authors combine bottom-up cost analysis of the GUI ' 98 cohort $(n=7525)$ and topdown analysis (applying international literature to various sources of national healthcare use and prevalence data) with a closed cohort simulation model for crossvalidation.

Raftery et al. (2012) study the individual and national direct and indirect cost of chronic non-cancer pain, and cost drivers, by collecting detailed information on healthcare use from 140 chronic pain patients randomly sampled from a national patient sample $(n=425)$ in the Prevalence, Impact and Cost of Chronic Non-Cancer Pain in Ireland (PRIME) study. Furthermore, Gannon et al. (2013) assess direct, indirect and informal costs related to severe chronic non-cancer pain in 100 patients attending a tertiary pain management clinic in the west of Ireland (Galway), representative of the larger chronic pain population in the PRIME study.

In the EPICure study, Petrou et al. (2009) assess 20062007-economic costs (hospital inpatient, day care and outpatient care; community health and social care, medication and (special) education services) and health utilities in eleven-year-old children born extremely preterm $(n=190)$ compared to matched term-born children $(n=$ 141 ) in Ireland and the UK, using parental questionnaires. In a succeeding study, Petrou et al. (2013) assess the economic outcome (cost and utilities described above) associated with neurodevelopmental impairment or disability at 11 years of age, comparing children born extremely preterm or at term in EPICure.

Richardson et al. (2012) collect data on medication use from adults aged 50 years and older in the nationally representative TILDA study $(n=8093)$ to estimate the prevalence and cost of polypharmacy in the ageing Irish population and infer potential cost savings if patients switched to generic medicines. 
Lastly, to estimate costs related to osteoarthritis and rheumatoid arthritis, Doherty and O'Neill (2014) compare self-reported healthcare use (primary care, inpatient and outpatient hospital care and A\&E) in adults aged 50 years and older with these arthritic conditions to healthcare use in the same age group without these conditions, collected in the nationally representative TILDA study $(n=8093)$.

\section{Costing approaches and data collection}

In terms of perspective, seven studies employed a societal (full [15, 17, 18, 21] and partial [22, 23, 27]), four a healthcare $[19,20,24,28]$, two a public sector $[25,26]$ and one a patient perspective [16]. Twelve studies employed a 'bottom-up' costing approach $[15,16,18-$ 23, 25-28] and two combined top-down and bottom-up $[17,24]$ (Table 2). Only four of the surveys presented in the inventory (Table 1) were employed amongst the identified literature.

For healthcare cost estimation, ten studies utilised data from a single survey [16, 19-23, 25-28]. Seven of these were dedicated ad-hoc surveys [16, 21-23, 25-27], four of which employed validated questionnaires (Client Service Receipt Inventory (CSRI) [21, 22, 27] and Resource Utilisation in Dementia Lite Instrument [23]). Of the other single-survey studies, two used TILDA [20, 28] and one employed data from the SLAN 2007 [19]. The remaining four studies employed data from a selection of surveys [18] and/or employed survey data alongside administrative data to determine utilisation $[15,17,24]$ and/or supplemented survey data with estimates from small-scale, local studies in the published literature [17, 24]. All survey data integrated in cost analysis were cross-sectional and collected retrospectively [15-18, 2028 , although longitudinal data may have been partially available in a few studies (e.g. TILDA, EPICure, GUI).

Survey data were most frequently used to assess costs of primary care, including general practitioner (GP) services, $(n=11)$ [15-24, 27], followed by costs of various hospital/residential care (including outpatient and emergency hospital services, $n=9$ ) $[15,18,19,21-23,25-27]$ and medications $(n=8)[16,17,21,22,25-28]$. Seven studies included specialist community services and other healthcare professionals $[15,17,22,23,25-27]$ and only two studies assessed costs in each of these areas [22, 27]. While there is variability in the range of healthcare services included when relying one data source (e.g. ranging from three to six) a greater range of services can be included when a specific validated instrument like CRSI is employed, albeit across a smaller sample size and studyspecific population [21-23, 27]. For example, when estimating the costs of osteoarthritis and rheumatoid arthritis, the choice of healthcare services considered was limited to those in the TILDA dataset [20].
For the various healthcare services assessed, recall periods differed across studies and sometimes within one study, ranging from one week to ten years or time since relapse or diagnosis; however most studies assessed a fixed period of six [23] or twelve months [18-20, 22, 24-27]. Where variable recall periods were assessed in one study, use of home help was assessed in the past week, medication use in the past month, diagnostic tests, outpatient visits and primary care in the past 6 months and inpatient hospital care in the past twelve months $[15,21]$. One study combined six- and twelve-month recall periods due to the structure of the underlying primary data [17]. Furthermore, Carney et al. (2018) and Fogarty et al. (2014) assessed costs for home appliances and mobility and other aids over the full duration of multiple sclerosis, and Carney et al. (2018) assessed various costs that occurred during the last multiple sclerosis relapse. Similarly, Ceilleachair et al. (2017) investigated out-of-pocket costs for the full duration of colorectal cancer disease and follow-up only. Richardson et al. (2012) assessed medication taken daily or weekly at the time of the interview and therefore did not specify a recall period.

\section{Unit prices}

Most studies $(n=11)$ used multiple sources to determine unit prices [15, 17-19, 21-27]. Most commonly, unit prices were informed by official national tariffs (national casemix/diagnostic-related groups) from Ireland $[15,17$, $18,21-24,27]$, the UK $[25,26]$, or national literature $[15,17-21,23,24]$. Studies also frequently relied on data from the Irish Central Statistics Office [15, 17, 18, 21$24]$, however this was mainly to determine unit prices related to productivity losses and pre-mature mortality [24]. Health professionals' expert opinions or other cost estimates directly retrieved from primary care services, hospitals and laboratory and finance departments were also used, as well as use of national salary scales or pharmaceutical price lists and databases $[15,19,21,22$, 24, 27]. Only few studies used information retrieved from social welfare services (e.g. national home scheme support) $[18,21]$, other company and service price lists $[15,21]$, patients' cost estimates $[16,21]$, or a specialised personal social service research unit (for assessment of community health and social service unit costs) $[25,26]$.

\section{Data quality and reliability}

When addressing study limitations, 13 studies referred to data issues [15-23, 25-28]. Concerns included absence of resource use data $[17,18,28]$, low response rates and potential for sampling bias and incomplete data, particularly in small dedicated ad-hoc surveys [16, 21,27 ], lack of suitable longitudinal/cross-sectional data to inform reliable bottom-up estimates [18, 22], reliance 
on dichotomous utilisation measures [19] and limited range of services [20] captured in population surveys. Issues surrounding the availability of data led researchers to employ a mix of top-down and bottom-up costing methodologies [17]. Where bottom-up only was employed, authors express concern regarding the generalisability of these results [22]. Additional concerns were highlighted relating to recall bias, which is inevitable when relying on retrospective patient surveys, rather than registry data $[15,16,21,27]$. This was raised particularly where surveys were completed on behalf of patients $[23,25,26]$. Risk of bias due to patient selfselection was mentioned in one study that collected information from a disease-specific patient sample [27]. As a side issue, several studies highlight the lack of reference costs for Ireland [17, 22, 23] and the fragmented nature of systems preventing linking [22].

Researchers addressed some of these limitations through sensitivity and sub-analyses and by providing estimated cost ranges, in addition to single figures presented in their main analysis [17-19, 21-24, 27]. These cost ranges were typically compared to international and, where available, national literature, and reasons for potential differences between study findings and the literature were frequently explored [1519, 21-28]. Patients with disabilities or comorbidities were explicitly excluded from some studies, or the analysis was adjusted for the added costs due to these conditions, so as not to overestimate disease-specific costs [17-19, 23]. Generally, researchers sought to use samples that were nationally or regionally representative of the wider population $[15,18,19,24]$, or of the patient group studied [15, 16, 22]. Where representation was not guaranteed, data were often weighted using national statistics or prevalence data before costs were extrapolated to the population level $[15,17-19,21,22,24,27,28]$. Additionally, bootstrapping was applied to address uncertainties related to small or unbalanced samples [17, 21, 23], and logarithmic transformations were applied to account for skewness of data [21, 22]. Where ad-hoc surveys were used, their design was often based on tested survey tools and included validated instruments (e.g. to assess disease status) $[15,17,21,23,25-27]$; some adhoc surveys were pre-piloted [22, 25-27] and mechanisms were used to increase participation (e.g. reminder letters) $[15,16,25,26]$ and completion (e.g. telephone interviews) [27]. Lastly, the interviewers of the TILDA data used in Richardson et al. (2012) asked participants to report and subsequently show interviewers the medication they were taking, to allow interviewers retrieve the details for all medication provided, for more accurate estimation of polypharmacy costs.

\section{Discussion}

This scoping review identified 14 studies from 2009 to 2019 using self-reported data on healthcare use or cost to assess direct healthcare costs in Ireland. Our search identified 27 surveys that provide self-reported data on health or healthcare use; only four pre-existing population surveys and six ad-hoc surveys were used to estimate costs according to our search criteria.

Despite the inclusion of self-reported healthcare use in many Irish datasets, this review demonstrates their limited application for the estimation of healthcare costs. One explanation could be related to the concern among some researchers that existing datasets are not fit for purpose, for example due to the limited choice of healthcare services for which data are collected, therefore necessitating the supplementation with published data and dedicated data collection. However, as this review reveals, such ad-hoc data collection efforts yield small samples, which are subject to selection bias. In fact, a number of identified nationally representative datasets with large sample sizes provide data on healthcare service utilisation, along with health status, demographic, socio-economic and lifestyle information. Many of these datasets consist of repeated cross-sectional or longitudinal survey waves, but have not been subject to cost assessments in Ireland to date. This could be linked to low awareness of these datasets among researchers and health economists (representation of the latter is relatively low in Ireland); difficulties in accessing datasets through publicly available sources and limited funding opportunities for analysing existing datasets. Research funding opportunities in Ireland tend to be biased towards collecting new data. Encouragingly, however, surveys designed and administered through research institutions (e.g. GUI, TILDA) are more likely to be utilised for cost analysis; suggesting that involving (health economic) researchers early in survey design could potentially increase the usability of population health surveys for cost analysis.

The studies included in this review assess the costs of multiple sclerosis, overweight and obesity, chronic noncancer pain, arthritis, dementia, very preterm birth, colorectal cancer and polypharmacy. This shows a tendency to non-acute, chronic conditions with a high prevalence that concern predominantly the older ages, or very young people and their future costs. It is questionable whether this implies sufficient resource coverage/ provision in acute care in Ireland, or may owe to the availability (and thus awareness) of large survey data particularly for the older ages (TILDA) and children (GUI). While all authors mention gaps in data availability, there is a considerably strong emphasis on the dearth in unit cost data particularly relating to older age [17] and demand for more accurate and reliable population-based 
information on health service utilisation, school/work productivity and psychosocial wellbeing of children [24].

Our survey inventory revealed mixed levels of data coverage. A number of population surveys convey a multitude of questions on use of various healthcare services, demographics, health-related risk and behaviour (GUI, SPHERE, the Irish Health Survey supplement to the QNHS and Healthy Ireland); making these surveys potentially more suitable for cost assessments relative to other surveys identified. Repeated surveys that are potentially still on-going and that collected data only for some healthcare services, e.g. primary care, should consider future extension to assess resource use more comprehensively, including use of outpatient hospital services (EU-SILC, Healthy Ireland, Household Budget Survey, Irish Health Survey-QNHS, SLAN and TILDA). This is in line with data limitations highlighted in studies that used some of these surveys for cost analysis. Particularly, these studies were able to investigate GP service use [18-20, 24], inpatient hospital care [18-20], outpatient hospital services [18-20], emergency department visits [20] and medication use [28]. In contrast studies using ad-hoc surveys additionally investigated various specialist services including imaging and laboratory investigations $[15,17,21-23,25-27]$ (rehabilitation, respite care and long-term care services [15, 21, 23], mental health services [22, 23, 27], alternative therapies $[22,27]$ and various equipment, home modification and transport costs (including ambulance care) $[15,16,21$, $22]$. Broad population surveys may accordingly benefit from the addition of an open-ended question where participants can detail the frequency and type of "other" healthcare services used during the study period.

Other (repeated) population surveys also would be more instrumental for comprehensive cost analysis if questionnaires were extended in future data collection. Namely, despite assessing healthcare use, the Household Budget Survey in its current form does not contain data on health status and lifestyle that would be needed for accurate cost analysis. Moreover, the Census and European Social Survey that are carried out routinely may be a good basis for collecting data on healthcare use and lifestyle, which is currently not the case. Similar to the estimation of healthcare use, most studies relied on a variety of sources to identify unit prices, suggesting that a central repository beyond the existing national casemix price list is needed. Some advances continue to be made in relation to this; however more healthcare services and consideration of methodologies still need to be incorporated [29].

While providing more detail on healthcare use, ad-hoc collection of data often led to relatively small, regional samples in comparison to studies using large datasets, thus impeding the studies' generalisability. Ad-hoc surveys sampled through national patient registers [15, 16] or from multiple healthcare service providers [27, 30] seemed to provide larger sample sizes than ad-hoc surveys sampled from individual healthcare service providers [21-23]; however effective sampling strategies are needed to reach representation and complete survey response [16]. Additionally, bootstrapping can be used to address concerns around distribution, e.g. to handle non-parametric data and small sample sizes [23]. Nevertheless, larger population surveys or centralised health system data appear preferential when representation cannot be otherwise reached.

Most studies collected information on resource use over a period of six to twelve months, with shorter periods or longer periods used for frequent use (e.g. medications and home care) or infrequent use (e.g. aids and home modifications), respectively. Studies identified these time periods as most appropriate for cost analysis and to involve a relatively low risk of recall bias based on previous research. However, all studies collected data retrospectively and risk of recall bias therefore cannot be fully ruled out. In response to this, use of diaries for prospective data collection was suggested for future studies [22], indicating the need for a more proactive approach to integrating healthcare use data in budgetary planning.

The challenges associated with lack of standardisation and interoperability of Irish health data is recognised and there are plans for reform [31, 32]. While details about the full implementation of an EHR system in Ireland are unknown [1], a data repository (eHealth Ireland Open Data Portal) providing information on health service use in Ireland has been established. However, this is limited to public health services only and currently contains a limited set of healthcare use data $[33,34]$. Overall, more digital solutions to manage patient data and connect health information within and across service providers and systems are being implemented. Examples include the National Medical Laboratory Information System [35], Digital Ambulance Project [36], Primary Care IT [37] and Epilepsy Lighthouse Project [38]. Most recently, the rollout of the national COVID-19 Vaccine Information System has highlighted the need for using unique patient identifiers in healthcare services, to ensure equitable, complete and safe access to vaccinations and to enable the system's interoperability within Europe through use of standardised vaccination certificates. Out of this need, the system draws on the existing Individual Health Identifiers that were developed on the basis of the Health Identifiers Act 2014, with the explicit aim to monitor and share personal details on health status, healthcare use and demographic information among healthcare providers and national institutions [39]. While this development shows promise to fill data gaps in the future, the 
implementation and integration of many individual initiatives and the national EHR system remain incomplete; thus the availability of integrated, comprehensive data for research purposes is likely to take time.

Accordingly, in Ireland and other jurisdictions with similar data restrictions, researchers continue to rely on self-reported data to conduct cost analysis. While researchers' concerns around recall bias and incomplete cost data indicate that self-reported data are sub-optimal for cost analysis, a recent study [40] demonstrated there is little difference in precision of cost estimates derived from registry data compared to well-designed health survey questionnaires. Another study highlighted that cost accuracy is primarily driven by the type of healthcare service studied [8]. Nevertheless, a review that compares cost studies using top-down, bottom-up or mixed costing approaches would be interesting in future research.

Meanwhile, acknowledging existing gaps that should be addressed in future data collection, the identified surveys and studies in this review can be used as goodpractice guides informing future survey development that enable comprehensive assessments of healthcare costs. Particularly, longitudinal and repeated crosssectional surveys, in their combined form, can provide representative estimates of healthcare use enabling cost comparisons across age groups and time. Future surveys need to be meaningful and fit for purpose; stakeholder approaches should be adopted where possible to enhance the value of the resulting data. Finally, to avoid future underutilisation of expensive population health surveys, generated data should be in line with the Findable, Accessible, Interoperable and Reusable (FAIR) Data Principles [41].

\section{Limitations}

Despite the use of a systematic search strategy, broad search criteria and the inclusion of both peer-reviewed and grey literature, it is possible that not all studies assessing healthcare costs using self-reported healthcare service use data were identified. While this review did not aim to retrieve a complete set of existing studies, cross-referencing and consistency checks using alternative search terms were employed to minimise the risk of bias. Similarly, despite using a broad search strategy, there is a risk that health surveys may be missing in the inventory. Therefore, we encourage other researchers to amend and publish an extended inventory if applicable. Furthermore, it should be noted that the analysis of underlying survey data in terms of their methodological quality is outside the scope of this research.

\section{Conclusion}

This scoping review identified 27 health surveys and 14 costing studies that used healthcare use or cost data from self-reported data, to assess direct healthcare costs in Ireland. The identified surveys that have the potential for analysing healthcare costs in Ireland are collated in an inventory. Researchers in and outside of Ireland can use this inventory to review data availability in the future. In the absence of integrated EHRs and unique patient identifiers in jurisdictions like Ireland, carefully designed healthcare surveys are useful tools for cost assessments; however barriers related to awareness, access and usability of these data must be considered.

\section{Abbreviations \\ CSRI: Client Service Receipt Inventory; EHR: Electronic Health Records; EU- SILC: European Union Statistics on Income and Living Conditions; FAIR: Findable, Accessible, Interoperable and Reusable; GUI: Growing Up in Ireland; SLAN: Survey of Lifestyle, Attitudes and Nutrition; TILDA: Irish Longitudinal Study on Ageing; ECAD: Enhancing Care in Alzheimer's Disease study; QNHS: Quarterly National Household Survey; PRIME: Prevalence, Impact and Cost of Chronic Pain; SPHERE: Secondary Prevention of Heart Disease in General Practice; GP: General Practitioner; PRISMA-ScR: Preferred Reporting Items for Systematic reviews and Meta-Analyses extension for Scoping Reviews}

\section{Supplementary Information}

The online version contains supplementary material available at https://doi. org/10.1186/s13561-021-00330-2.

Additional file 1: Table S1. Literature search terms and result. Figure S1. Flow chart of search process and results.

Acknowledgements

Not applicable.

Authors' contributions

AM, SBJ and JS contributed to the conception and design of the work; SBJ and GV contributed to the acquisition, analysis, and interpretation of data;

SBJ and AM drafted the work. All authors commented on drafts and approved the version to be published.

Funding

No funding supports were received for this research.

Availability of data and materials

All data generated or analysed during this study are included in this published article.

\section{Declarations}

Ethics approval and consent to participate

Not applicable.

Consent for publication

Not applicable.

Competing interests

Authors have no conflicts to declare.

Author details

${ }^{1}$ Healthcare Outcomes Research Centre, Royal College of Surgeons in Ireland, Dublin, County Dublin, Ireland. ${ }^{2}$ Department of Economics, Cork University Business School, University College Cork, Cork, County Cork, Ireland. 
Received: 16 February 2021 Accepted: 11 August 2021

\section{Published online: 16 September 2021}

\section{References}

1. Oderkirk J. Readiness of electronic health record systems to contribute to national health information and research. Findings of the 2016 OECD HCQI study of electronic health record system development and data use. OECD. 2017.

2. eHealth Ireland. Latest Progress: Health Services Executive; 2020 [Available from: https://www.ehealthireland.ie/A2I-HIDs-Programme/Individual-HealthIdentifier-|HI-/Latest-Progress/.

3. eHealth Ireland. Individual Health Identifier $(\mathrm{IHI})$ Frequently asked Questions: Health Service Executive; 2020 [Available from: https://www.ehealthireland. ie/A2I-HIDs-Programme/Individual-Health-Identifier-IHI-/Information-for-thePublic/IHI-FAQs-Public.pdf.

4. Hammond WE, Bailey C, Boucher P, Spohr M, Whitaker P. Connecting information to improve health. Health Aff. 2010;29(2):284-8. https://doi. org/10.1377/hlthaff.2009.0903.

5. Frappier J, Tremblay G, Charny M, Cloutier LM. Costing bias in economic evaluations. J Med Econ. 2015;18(8):596-9. https://doi.org/10.3111/13 696998.2015.1033423.

6. Lette M, Bemelmans WJE, Breda J, Slobbe LCJ, Dias J, Boshuizen HC. Health care costs attributable to overweight calculated in a standardized way for three European countries. Eur J Health Econ. 2016;17(1):61-9. https:/doi. org/10.1007/s10198-014-0655-8.

7. GenericØdegård R, Balanda KP, Bjørnelv GW, Halsteinli V, Jabakhanji SB, Sonntag D, et al. Big data and simulation modelling of obesity: the need for greater international collaboration and coordination. Eur Congress Obesity. 2021.

8. Chapko MK, Liu C-F, Perkins M, Li Y-F, Fortney JC, Maciejewski ML. Equivalence of two healthcare costing methods: bottom-up and top-down. Health Econ. 2009;18(10):1188-201. https://doi.org/10.1002/hec.1422.

9. Špacírová Z, Epstein D, García-Mochón L, Rovira J. Olry de Labry Lima a, Espín J. a general framework for classifying costing methods for economic evaluation of health care. Eur J Health Econ. 2020;21(4):529-42. https://doi. org/10.1007/s10198-019-01157-9.

10. Munn Z, Peters MDJ, Stern C, Tufanaru C, McArthur A, Aromataris E. Systematic review or scoping review? Guidance for authors when choosing between a systematic or scoping review approach. BMC Med Res Methodol. 2018;18(1):143. https://doi.org/10.1186/s12874-018-0611-x.

11. Arksey H, O'Malley L. Scoping studies: towards a methodological framework. Int J Soc Res Methodol. 2005;8(1):19-32. https://doi.org/10.1080/1364557032 000119616.

12. Drummond MF, Sculpher MJ, Torrance GW, O'Brien BJ, Stoddart GL. Methods for the economic evaluation of health care programmes. Oxford: Oxford University Press; 2005.

13. Tricco AC, Lillie E, Zarin W, O'Brien KK, Colquhoun H, Levac D, et al. PRISMA extension for scoping reviews (PRISMA-SCR): checklist and explanation. Ann Intern Med. 2018;169(7):467-73. https://doi.org/10.7326/m18-0850.

14. Peters MD, Godfrey CM, Khalil H, Mclnerney P, Parker D, Soares CB. Guidance for conducting systematic scoping reviews. Int J Evid-based Healthcare. 2015;13(3):141-6. https://doi.org/10.1097/xeb.0000000000000050.

15. Carney P, O'Boyle D, Larkin A, McGuigan C, O'Rourke K. Societal costs of multiple sclerosis in Ireland. J Med Econ. 2018;21(5):425-37. https://doi.org/1 0.1080/13696998.2018.1427100

16. Ceilleachair AO, Hanly P, Skally M, O'Leary E, O'Neill C, Fitzpatrick P, et al. Counting the cost of cancer: out-of-pocket payments made by colorectal cancer survivors. Supp Care Cancer. 2017;25(9):2733-41. https://doi.org/10.1 007/s00520-017-3683-y.

17. Connolly S, Gillespie P, O'Shea E, Cahill S, Pierce M. Estimating the economic and social costs of dementia in Ireland. Dementia (London). 2014;13(1):5-22. https://doi.org/10.1177/1471301212442453.

18. Dee A, Callinan A, Doherty E, O'Neill C, McVeigh T, Sweeney MR, et al. Overweight and obesity on the island of Ireland: an estimation of costs. BMJ Open. 2015;5(3):e006189. https://doi.org/10.1136/bmjopen-2014-0061 89.

19. Doherty E, Dee A, O'Neill C. Estimating the amount of overweight and obesity related health-care use in the Republic of Ireland using SLÁN data. Econ Soc Rev. 2013;43(2):227-50 https://www.esr.ie/article/view/48.

20. Doherty E, O'Neill C. Estimating the health-care usage associated with osteoarthritis and rheumatoid arthritis in an older adult population in
Ireland. J Public Health (Oxford, England). 2014;36(3):504-10. https://doi. org/10.1093/pubmed/fdt097.

21. Fogarty E, Walsh C, McGuigan C, Tubridy N, Barry M. Direct and indirect economic consequences of multiple sclerosis in Ireland. Appl Health Econ Health Policy. 2014;12(6):635-45. https://doi.org/10.1007/s40258-014-0128-3.

22. Gannon B, Finn DP, O'Gorman D, Ruane N, McGuire BE. The cost of chronic pain: an analysis of a regional pain management service in Ireland. Pain Med (Malden, Mass). 2013;14(10):1518-28. https://doi.org/10.1111/pme.12202.

23. Gillespie P, O'Shea E, Cullinan J, Lacey L, Gallagher D, Ni MA. The effects of dependence and function on costs of care for Alzheimer's disease and mild cognitive impairment in Ireland. Int J Geriatr Psychiatry. 2013;28(3):256-64. https://doi.org/10.1002/gps.3819.

24. Perry IJ, Millar SR, Balanda KP, Dee A, Bergin D, Carter L, et al. What are the estimated costs of childhood overweight and obesity on the island of Ireland?Cork; 2017.

25. Petrou S, Abangma G, Johnson S, Wolke D, Marlow N. Costs and health utilities associated with extremely preterm birth: evidence from the EPICure study. Value Health. 2009;12(8):1124-34. https://doi.org/10.1111/j.1524-4 733.2009.00580.x.

26. Petrou S, Johnson S, Wolke D, Marlow N. The association between neurodevelopmental disability and economic outcomes during midchildhood. Child Care Health Dev. 2013;39(3):345-57. https://doi.org/1 0.1111/j.1365-2214.2012.01368.x.

27. Raftery MN, Ryan P, Normand C, Murphy AW, de la Harpe D, McGuire BE. The economic cost of chronic noncancer pain in Ireland: results from the PRIME study, part 2. J Pain. 2012;13(2):139-45. https://doi.org/10.1016/j.jpain.2011.10.004.

28. Richardson K, Moore PV, Peklar J, Galvin R, Bennett K, Kenny RA. Polypharmacy in adults over 50 in Ireland: opportunities for cost savings and improved healthcare. Dublin: Trinity College Dublin; 2012.

29. Smith S, Jiang J, Normand C, O'Neill C. Unit costs for non-acute care in Ireland 2016?2019 [version 1; peer review: awaiting peer review]. HRB Open Res. 2021;4(39). https://doi.org/10.12688/hrbopenres.13256.1.

30. Raftery MN, Sarma K, Murphy AW, De la Harpe D, Normand C, McGuire BE. Chronic pain in the Republic of Ireland--community prevalence, psychosocial profile and predictors of pain-related disability: results from the prevalence, impact and cost of chronic pain (PRIME) study, part 1. Pain. 2011;152(5):1096-103. https://doi.org/10.1016/j.pain.2011.01.019.

31. Health Information and Quality Authority. Developing eHealth interoperability standards for Ireland. Statement of outcomes from the public consultation; 2017.

32. Committee on the Future of Healthcare. Sláintecare report houses of the Oireachtas; 2017.

33. eHealth Ireland. Open data for health: health services executive; 2020. Available from: https://www.ehealthireland.ie/Strategic-Programmes/OpenData-for-Health/

34. eHealth Ireland. Ireland's health data at you fingertips: health services executive; 2020. Available from: https://data.ehealthireland.ie/

35. eHealth Ireland. National Medical Laboratory Information System (MedLIS): Health Service Executive; 2020 [Available from: https:/www.ehealthireland.ie/StrategicProgrammes/National-Medical-Laboratory-Information-System-MedLIS-/.

36. eHealth Ireland. HSE Ambulances Go Digital Digital Ambulance Project 2019: Health Service Executive; 2020 [Available from: https:/www.ehealthireland.ie/ News-Media/News-Archive/2019/HSE-Ambulances-Go-Digital-html.

37. eHealth Ireland. Primary Care IT: Health Services Executive; 2020 [Available from: https://www.ehealthireland.ie/Strategic-Programmes/Primary-Care-IT/.

38. eHealth Ireland. Epilepsy Lighthouse Project Update 20182018 [Available from: https://www.ehealthireland.ie/News-Media/News-Archive/2018/ Epilepsy-Lighthouse-Project-Update-2018.html.

39. Government of Ireland, Department of Health, Health Service Executive. Vaccine Information System for COVID-19 Vaccination Programme - Data Protection Impact Assessment Version 1.8. 2021 22/04/2021.

40. Sorensen J, Jabakhanji SB, McElroy B, Valentelyte G, Burke LA, Murphy A. Benchmarking cost models in a data desert. Nordic health economic study group. Reykjavik: NHESG; 2019.

41. Wilkinson MD, Dumontier M, Aalbersberg IJ, Appleton G, Axton M, Baak A, et al. The FAIR guiding principles for scientific data management and stewardship. Sci Data. 2016;3(1):160018. https://doi.org/10.1038/sdata.2016.18.

\section{Publisher's Note}

Springer Nature remains neutral with regard to jurisdictional claims in published maps and institutional affiliations. 